\title{
Creation of a Sudden Cardiac Arrest Safety Net
}

\author{
Mary McCormack, DNP, APRN, FNP-C \\ Molloy College \\ Carole Zarcone, DNP, APRN, ANP-C \\ Molloy College \\ Kendra Hoepper, DNP, APRN, PNP-BC \\ Long Island University \\ Pamela Watters, PhD, MSS, MSCS \\ Molloy College
}

\begin{abstract}
Background: More than 350,000 episodes of out-of-hospital cardiac arrest (OHCA) occur annually in the United States, with less than half of the victims receiving bystander cardiopulmonary resuscitation (CPR). Provision of bystander CPR has been noted to increase survival rates two to three-fold. However, bystander CPR is initiated in less than $50 \%$ of out of OHCA episodes in the United States.

Aim: The purpose of this pilot study was to create a sudden cardiac arrest safety net on a college campus. The American Heart Association (AHA) CPR in Schools Program ${ }^{\odot}$ was provided to college students, athletes, faculty and staff.

Methods: A multi-group educational intervention with a pre- and post-test design.

Results: Participant knowledge level of CPR and automated external defibrillator (AED) use significantly improved on the post test. Additionally, after attending the sessions participants reported an increase in comfort level performing CPR and improved knowledge of the locations of the AEDs on campus. As a result of the program, nine additional AEDs have been placed in high-traffic areas on campus.

Conclusion: Empowering laypersons with the skills and knowledge to respond to potential episodes of OHCA are integral steps towards improving patient outcomes.
\end{abstract}

Submitted 10 October 2020: accepted 27 November 2020

Keywords: out of hospital cardiac arrest, cardiopulmonary resuscitation, automated external defibrillator, hands-only CPR

Nationally, more than 350,000 episodes of out-of-hospital cardiac arrest (OHCA) occur annually with less than half of the victims receiving bystander cardiopulmonary resuscitation (CPR; Mozzafarian et al., 2016). Sudden cardiac arrest (SCA) occurs as a result of an electrical malfunction of the heart and is characterized by an abrupt loss of consciousness and collapse. Survival and future neurologic status post cardiac arrest are directly dependent upon how quickly the victim receives emergency medical treatment (Anderson et al., 2014; Drezner, 2009; Institute of Medicine, 2012; Institute of Medicine, 2015; Rega \& Fink, 2015). Approximately 38\% of OHCA episodes are witnessed by a 
layperson and initiation of bystander CPR has been noted to improve survival rates two to three-fold (Institute of Medicine, 2015; Virani et al., 2020).

The national survival rate for OHCA is approximately 10.4\%, with great variability noted between regions (Virani et al., 2020). Over the past decade, the placement of automated external defibrillators (AEDs) in public venues has been encouraged globally, yet less than 3\% of the American public have received CPR and AED training (Anderson, et al., 2014). Although cardiac arrest can occur anywhere, it has been noted that the majority of OHCA episodes occur in the home (69.8\%), followed by public settings (18.8\%) and (11.5\%) in nursing homes (Virani et al., 2020).

Geographic and socioeconomic disparities have been correlated with low levels of CPR training and poor OHCA outcomes (Sassoon, Haukoos, Eigel, \& Magid, 2014). In communities with low median household incomes, there are greater incidences of OHCA, provision of bystander CPR is less likely, and mortality rates are higher when compared to communities with higher median household incomes (Abdulhay et al., 2019). Communities reporting a large percentage of laypersons trained in CPR, such as King County, Seattle, where $75 \%$ of the community is trained in CPR, boast SCA survival rates upwards of 50\% (Resuscitation Academy, 2014).

Since the inception of closed chest CPR in the acute setting in the 1970's, there have been great strides made towards providing community CPR education. However, barriers such as fear of disease transmission, fear of causing harm, and concerns regarding legality have prevented bystanders from providing CPR. Implementation of CPR and AED educational programs have been identified by both the Institute of Medicine and the American Heart Association (AHA) as key strategies for improving OHCA outcomes (Institute of Medicine 2015; Neumar et al., 2015). The AHA's Hands Only CPR ${ }^{\odot}$ campaign has simplified CPR education, as "mouth to mouth" resuscitation is no longer required of the rescuer. In addition, Good Samaritan legislation has been enacted in all 50 states, providing legal protection to laypersons willing to help OHCA victims.

In a medically advanced society with easy access to technology, nationwide OHCA survival rates of $10.4 \%$ are incomprehensible (Virani et al., 2020). Empowering lay persons with lifesaving skills to assist victims of OHCA is of utmost importance and is a societal obligation. Knowledge is power, and implementation of CPR and AED programs provide individuals with the power to change lives. The purpose of this educational intervention was to provide college students, athletes, faculty, and staff with Hands Only $\mathrm{CPR}^{\odot}$ education, with the overarching goal to create a "Sudden Cardiac Arrest Safety Net" on the campus.

\section{METHODS}

This was a multi-group educational intervention with a pre- and post-test design. The location for the CPR and AED program was a liberal arts college in Nassau County, NY. Participants were recruited via email and each session lasted approximately 60 minutes. A convenience sample of college students, athletes, faculty, and staff students were invited to participate in the AHA's CPR in Schools ${ }^{\mathcal{O}}$ program. The CPR in Schools ${ }^{\odot}$ program can be implemented by a course facilitator and does not require a certified basic life support instructor. The instructional kit contains all the necessary materials for students to learn about and practice Hands Only $\mathrm{CPR}^{\odot}$. In addition, AED use was demonstrated by the course facilitators with an AED trainer.

Participants were required to complete a brief demographic questionnaire, as well as a pre and post-test which were obtained through purchasing the AHA CPR in Schools ${ }^{\mathcal{O}}$ kit. There were six CPR and AED knowledge questions, including one question addressing comfort related to performing CPR, and an additional question regarding 
knowledge of the AED locations on campus. Participants were encouraged to complete the questionnaire and tests electronically via google forms, paper copies were also available.

Descriptive and comparative (paired $t$-test) statistical analyses were performed using IBM SPSS statistical software Version 23

\section{RESULTS}

Descriptive statistical analysis of the sample $(n=161)$ revealed the majority of participants were white $(88.8 \%)$ females $(91.9 \%)$. There was good representation from both the student body $(54 \%)$ as well as the faculty and staff $(46 \%)$. A paired samples $t$-test was conducted to compare knowledge level of CPR and AED use in participants before and after implementation of the AHA CPR in Schools ${ }^{\odot}$ program. There was a significant difference, $t(160)=24.11, p<$ .001 , between pre-test $(M=2.65 ; S D=1.64)$ and post-test scores $(M=5.72 ; S D=0.46)$. Statistical analysis further revealed an extremely large effect size (Cohen's $d=1.873$ ), indicating that the program probably helped the learners improve their scores by almost 2 standard deviations (Table 1 ).

A paired samples $t$-test revealed there was a significant difference, $t(156)=17.50, p<0.001$, in comfort level in performing CPR if participants witnessed a cardiac arrest. Prior to partaking in the program, only $31 \%$ of participants reported that they would feel comfortable performing CPR if they witnessed a cardiac arrest. Whereas after participating in the CPR in Schools program ${ }^{\circ}, 97 \%$ of participants reported they would feel comfortable performing CPR if they witnessed a cardiac arrest. Statistical analysis also showed a large effect size (Cohen's $d=1.38$; Table 1).

An additional paired samples $t$-test was performed comparing participant knowledge of the locations of AEDs on campus before and after the session. Results were coded as " 1 " if participants were aware of AED locations and " 0 " if they were unaware of AED locations. There was a significant difference $t(158)=27.19, p<.001$ in the pre-test $(M=0.164, S D=0.371)$ and post-test score $(M=0.987, S D=0.112)$, which strongly supports that participants were more aware of the location of the AEDs on campus after the session. Again, the effect size was very large (Cohen's $d$ $=2.15$; See Table 1).

These results support that implementation of the AHA CPR in Schools ${ }^{\odot}$ program improves both knowledge and comfort level of performing CPR and AED use in this group of college students, athletes, faculty and staff. 
Table 1

Pre-Test and Post-Test Scores

\begin{tabular}{|c|c|c|c|c|c|c|c|}
\hline Measure & & Pre-Test & & $\begin{array}{l}\text { Post- } \\
\text { Test }\end{array}$ & & $\begin{array}{l}\text { Effect Size } \\
\text { (Cohen's } \\
\text { d) }\end{array}$ & $P$ \\
\hline & $n$ & Mean & $\begin{array}{l}\text { Std. } \\
\text { Dev }\end{array}$ & Mean & Std. Dev & & \\
\hline $\begin{array}{l}\text { AHA CPR } \\
\text { in Schools }\end{array}$ & 161 & 2.65 & 1.64 & 5.73 & 0.46 & 1.87 & $<0.001$ \\
\hline $\begin{array}{l}\text { Comfort } \\
\text { level } \\
\text { performing } \\
\text { CPR if } \\
\text { witnessed } \\
\text { cardiac } \\
\text { arrest }\end{array}$ & $157^{*}$ & 0.31 & 0.46 & 0.97 & 0.18 & 1.38 & $<0.001$ \\
\hline $\begin{array}{l}\text { Knowledge } \\
\text { of location } \\
\text { of AEDs } \\
\text { on campus }\end{array}$ & $159^{*}$ & 0.16 & 0.37 & 0.99 & 0.11 & 2.15 & $<0.001$ \\
\hline
\end{tabular}

Note. ${ }^{*}$ Missing data

\section{DISCUSSION}

Nationwide OHCA survival rates are poor, with urban areas such as Chicago, Detroit, and New York City reporting even lower survival rates of < 5\% (Abrams, McNally, Ong, Moyer, \& Dryer, 2013; Institute of Medicine, 2015; Resuscitation Academy, 2014). The best evidence suggests that providing community CPR and AED education is a proven and cost-effective means towards improving OHCA outcomes (Abrams et al., 2013; Resuscitation Academy, 2014). Communities of low socioeconomic status are disproportionately affected by OHCA and an emphasis should be placed on improving layperson CPR training rates in these neighborhoods.

Curriculum changes and alternative learning environments have made it easier to implement CPR programs in the community setting. Certified instructors are no longer required to teach layperson CPR and most programs are offered free of charge. Programs such as the AHA's CPR Anytime ${ }^{\odot}$ and CPR in Schools ${ }^{\odot}$ can be implemented by school teachers and laypersons. While the human toll is evident, the financial impact on the United States health care system is also significant. The average cost of treating victims of OHCA has been estimated as $\$ 102,017$ per person, placing an annual financial burden of $\$ 33$ billion dollars on the United States health care system (Kida \& Ichinose, 2014). 
The evidence continues to demonstrate that communities of low socioeconomic status are disproportionately affected by a multitude of health disparities. The next phase of the project is underway and involves an interdisciplinary team of nursing and allied health faculty and students providing CPR and cardiovascular health education in high-risk communities. The project team has been continuously collaborating with the public safety department at the college, and since the implementation of the program, an additional nine AEDs have been placed in high-traffic areas on campus. The project team is currently working towards obtaining the Heart Safe Community certification for the college campus from the Citizen CPR Foundation.

\section{LIMITATIONS}

The majority of the AHA CPR in Schools ${ }^{\odot}$ sessions were offered during National CPR \& AED Awareness week in June 2019 when most of the students and faculty were already on summer vacation. A larger sample size may have resulted if the program had been offered to the entire campus community during the spring or fall semesters. Lastly, participants choosing to enroll in the sessions may be more altruistic and more committed to learning CPR than those who chose not to participate. 


\section{REFERENCES}

Abrams, H. C., McNally, B., Ong, M., Moyer, P. H., \& Dyer, K. S. (2013). A composite model of survival from out-of-hospital cardiac arrest using the Cardiac Arrest Registry to Enhance Survival (CARES). Resuscitation, 84(8), 1093-1098. https://doi.org/10.1016/j.resuscitation.2013.03.030

Abdulhay, N., Konstantinos, T., McGovern, S., Hewitt, N., Bhardwaj, A., Buckler, D., Abella, B. (2019). Socioeconomic disparities in layperson CPR training within a large U.S. city. Resuscitation, 141, 1318. https://doi.org/10.1016/j.resuscitation.2019.05.038

Anderson, M. L., Cox, M., Al-Khatib, S. M., Nichol, G., Thomas, K. L., Chan, P. S., Peterson, E. D. (2014). Cardiopulmonary Resuscitation Training Rates in the United States. JAMA Internal Medicine, 174(2), 194 201. https://doi.org/10.1001/jamainternmed.2013.11320

Drezner, J. (2009). Preparing for sudden cardiac arrest - the essential role of automatic external defibrillators in athletic medicine: A critical review. British Journal of Sports Medicine, 43, 702-707. https://doi.org/10.1136/bjsm.2008.054890

Institute of Medicine. (2015). Strategies to improve cardiac arrest survival: A time to act. Washington, DC: The National Academic Press. Retrieved from https://www.ncbi.nlm.nih.gov/books/NBK321501/

Institute of Medicine (2012). Crisis standards of care: A systems framework for catastrophic disaster response. Washington, DC: The National Academic Press. https://doi.org/10.17226/13351

Kida, K., \& Ichinose, F. (2014). Preventing ischemic brain injury after sudden cardiac arrest using NO inhalation. Critical Care, 18(2), 212. https://doi.org/10.1186/cc13779

Mozzaffarian, D., Benjamin, E., Go, A., Arnett, D., Blaha, M., Cushman, M., ...Turner, M. (2016). Executive summary: Heart disease and stroke statistics -2016 update. Circulation, 133(4), e 38-e360. https://doi.org/10.1161/CIR.0000000000000366

Neumar, R. W., Eigel, B., Callaway, C. W., Estes, M., Gollis, J. G. Kleinman, M. E., ... Sendelback, S. (2015). American Heart Association response to the 2015 Institute of Medicine report on strategies to improve cardiac arrest survival. Circulation, 132, 1049-1070. https://doi.org/10.1161/CIR.0000000000000233

Rega, P., \& Fink, B. (2015). AED education: A dilemma for public health and a challenge for critical care specialists. Journal of Intensive and Critical Care, 1(1), 1-4. https://doi.org/10.21767/2471-8505.10009

Resuscitation Academy. (2014). Strategies to improve survival from cardiac arrest: An evidence-based analysis. Seattle, WA: Resuscitation Academy. Retrieved from https://www.resuscitationacademy.org/downloads/RA-35Strategies-to-Improve-CA-Survival.pdf

Sassoon, C., Haukoos, J., Eigel, B. \& Magid, D. (2014). The HANDDS Program: A systematic approach to addressing disparities in the provision of bystander cardiopulmonary resuscitation. Academic Emergency Medicine, 21, 1042-1049. https://doi.org/10.1111/acem.12455

Virani, S., Alonso, A., Benjamin, E., Bittencort, M., Callaway, C., Carson, A., ... Van Wagner, L. (2020). Heart disease and stroke statistics-2020 Update: A report from the American Heart Association. Circulation, 141, e139-e596. https://doi.org/10.1161/CIR.0000000000000757 
Address author correspondence to:

Mary McCormack, DNP, APRN, FNP-C

Assistant Professor

Molloy College, Barbara Hagan School of Nursing \& Health Sciences

1000 Hempstead Avenue

Rockville Centre, NY 11570

mmccormack1@molloy.edu

Carole Zarcone, DNP, APRN, ANP-C

Assistant Professor

Molloy College, Barbara Hagan School of Nursing \& Health Sciences

1000 Hempstead Avenue

Rockville Centre, NY 11570

czarcone@molloy.edu

Kendra Hoepper, DNP, APRN, PNP-BC

Assistant Professor

Director Undergraduate Nursing Program

Long Island University, Post School of Health Professions \& Nursing

720 Northern Blvd

Brookville, NY 11548

kendra.hoepper@liu.edu

Pamela Watters, PhD, MSS, MSCS

Statistical Support Consultant

Office of Graduate Academic Affairs

Molloy College

1000 Hempstead Avenue

Rockville Centre, NY 11570

pwatters@molloy.edu

\section{Project Funding}

Nurse Practitioner Healthcare Foundation/Astellas Heart Health Thorough the Life Span Award

Molloy College Sigma Theta Tau: Epsilon Kappa Chapter

Molloy College Faculty Research Grant

\section{Author's Note and Acknowledgements}

The authors would like to thank the students, faculty and staff at Molloy College who participated in the research study. 